\title{
Perovskite Solar Cell: A Game Changer in Future Solar Power
}

\author{
Nam-Gyu Park \\ School of Chemical Engineering, Sungkyunkwan University, Korea \\ (E-mail: npark@skku.edu)
}

\begin{abstract}
Since the first report on the $9.7 \%$ efficient and 500 h-stable solid-state perovskite solar cell (PSC) in 2012 based on methylammonium lead iodide, following two seed reports on perovskite-sensitized liquid junction solar cells in 2009 and 2011, perovskite photovoltaics have been surged swiftly due to high power conversion efficiency (PCE) obtainable via facile fabrication procedure. As a result, a PCE of $25.5 \%$ was recorded in 2020. According to Web of Science, number of publications on PSCs increases exponentially since 2012, leading to the accumulated publications of more than 23,400 as of October 16, 2021. PSC is regarded as a game changer in photovoltaics because of low-cost and high efficiency surpassing the conventional high efficiency thin film technologies. High photovoltaic performance was realized by compositional engineering, device architecture and fabrication methodologies for the past 10 years. Toward theoretical efficiency over $30 \%$ and commercialization of PSCs, further studies on recombination and scalable technologies are required for next 10 years. In this talk, scientific and technological approaches for high efficiency and large-area coating are discussed. For high efficiency, not only perovskite materials and coatings but also interfacial engineering via additive and post-treatment is of importance. For upscaling PSCs, precursor formulation and coating methods are critical in determining photovoltaic performance. Stability issue will be also discussed and methodologies to improve stability are suggested.
\end{abstract}

